\title{
Ocular Manifestations of Patients With Cutaneous Rosacea With and Without Demodex Infection
}

\author{
Yesim Akpinar Kara, MD; Feyza Çalış, MD; İrem Burçak Gürel, MD
}

\section{PRACTICE POINTS}

- Rosacea is a common chronic inflammatory skin disease of the central facial skin and is of unknown origin. Patients with ocular rosacea may report dryness, itching, and photophobia.

- Demodex infestation may increase cutaneous or ocular inflammation in rosacea.
Rosacea is a chronic progressive disease that causes inflammation on the skin and the ocular surface. This study aimed to evaluate the effects of Demodex mites on clinical findings associated with rosacea. Sixty patients who were newly diagnosed with rosacea in the dermatology outpatient clinic underwent superficial skin biopsy to determine the density of Demodex species. The patients were evaluated as Demodex positive $(n=30)$ or Demodex negative $(n=30)$ based on the species density. The 60 patients were examined in the ophthalmology outpatient clinic; a total of 120 eyes underwent tear breakup time (TBUT) and Schirmer tests and were examined for meibomitis and blepharitis findings. The demographic characteristics and ocular findings of both groups were recorded and statistically compared. We found that Demodex mite-related inflammation in rosacea does not significantly affect clinical ocular surface findings and that Demodex positivity is significantly associated with papulopustular rosacea $(\mathrm{PPR})(P=.003)$.

Cutis. 2021;108:46-50.

A cne rosacea is a chronic inflammatory disease that may affect the facial skin, eyes, and eyelids. ${ }^{1}$ It is characterized by transient or persistent flushing, facial erythema, and telangiectases, generally located on the central portion of the face, and may progress to papules and pustules. ${ }^{2,3}$ At the late stage of the disease, dermal edema or fibroplasia and sebaceous gland hypertrophy may cause phymatous alterations in the skin. In 2004, the National Rosacea Society Expert Committee developed a classification system for rosacea to standardize subtypes and variants that has since been widely accepted and continues to aid in research and epidemiologic studies. ${ }^{4}$ The committee defined 4 subtypes based on clinical characteristics: erythematotelangiectatic (ETR), papulopustular (PPR), phymatous, and ocular rosacea. ${ }^{2,3}$

Ocular rosacea may accompany mild, moderate, and severe dermatologic disease or may occur in the absence of diagnostic skin disease. ${ }^{5}$ Ocular signs include eyelid margin telangiectasia, spade-shaped infiltrates in the cornea, scleritis, and sclerokeratitis. Common symptoms include burning, stinging, light sensitivity, and foreignbody sensation. Ocular signs commonly seen in rosacea are meibomian gland dysfunction characterized by inspissation and inflammation of the meibomian glands (chalazia), conjunctivitis, honey crust and cylindrical collarette accumulation at the base of the eyelashes, irregularity of the eyelid margin architecture, and evaporative tear dysfunction. 5,6

The physiopathology of rosacea is still unknown. Potential factors include genetic predisposition, abnormal inflammation, vascular dysfunction, and involvement of several microbial agents, such as commensal Demodex mites. The number of Demodex mites on normal skin flora is less than $5 / \mathrm{cm}^{2}$; however, the increased vascular dilation and capillary permeability associated with rosacea that result from sunlight and heat exposure increase the density of Demodex folliculorum. ${ }^{7}$ Elevated Demodex mite

Drs. Kara and Çaliş are from Koru Hospital, Ankara, Turkey. Dr. Kara is from the Department of Dermatology, and Dr. Çaliş is from the Department of Ophthalmology. Dr. Gürel is from the Department of Ophthalmology, 29 Mayıs State Hospital, Ankara.

The authors report no conflict of interest.

Correspondence: Yesim Akpinar Kara, MD, 1428.sk No:16/8, Cukurambar, Ankara, Turkey 06520 (yesim_akpinar@yahoo.com). doi:10.12788/cutis.0289 
density has been observed in the lumens of the sebaceous follicles in patients with rosacea. However, because the severity of the clinical manifestations of the disease is not directly associated with the density of $D$ folliculorum, it generally is accepted that $D$ folliculorum is not a pathogenetic but rather an exacerbating factor. ${ }^{8}$ It has been reported that this species of mite is mostly found on the face and around the eyelashes and scalp of patients and that it can cause ocular surface inflammation. ${ }^{8}$

Most studies have researched ocular manifestations of rosacea but not ocular involvement in rosacea patients with and without Demodex mite infestation. In our study, we sought to compare the ocular surface, meibomian gland characteristics, and tear film abnormalities among patients with cutaneous rosacea with and without Demodex infestation.

\section{Materials and Methods}

We conducted a retrospective study of 60 patients with cutaneous rosacea. This study was approved by the ethics committee of the local hospital (2018/002-003), and all patients provided verbal and written informed consent before participating in the study. The study was carried out according to the guidelines of the Declaration of Helsinki.

Patient Selection and Evaluation-Patients diagnosed with rosacea by a dermatologist within 6 months were included in the study. Diagnosis of the disease was made after a detailed anamnesis and dermatologic examination. Rosacea was diagnosed if patients had an itching sensation, erythema and/or erythema attacks, and papules and pustules, and fulfilled the diagnostic criteria according to the National Rosacea Society. The skin disease was classified according to the subtypes as ETR, PPR, phymatous rosacea, or ocular rosacea.

The standard skin surface biopsy method was used in 60 patients for detecting Demodex density. When more than 5 mites were detected per square centimeter, the result was recorded as positive. Thirty consecutive, newly diagnosed patients with cutaneous acne rosacea with Demodex infestation and 30 consecutive, newly diagnosed sex- and age-matched patients with acne rosacea without Demodex infestation admitted to the dermatology outpatient clinic were included to this study. The patients who did not have any known dermatologic, systemic, or ocular diseases were included in the study. Patients who met any of the following criteria were excluded from the study: prior anti-inflammatory topical and/or systemic treatment for rosacea during the last 3 months, contact lens wear, eyelid surgery, or autoimmune disease requiring treatment.

Microscopic Demodex Examination-Demodex count was determined using a standardized skin surface biopsy, which is a noninvasive method. Every patient gave samples from the cheeks. This biopsy was repeated from the same site. A drop of cyanoacrylate was placed on a clean slide, pressed against a skin lesion, held in place for 1 minute, and removed. The obtained samples were evaluated under a light microscope (Nikon E200) with oil immersion. When more than 5 mites were detected per square centimeter, the result was recorded as positive.

Ophthalmologic Examination-A complete ophthalmologic examination including visual acuity assessment, standardized slit lamp examination, and fundus examination was done for all patients. Ocular rosacea was diagnosed on detection of 1 or more of the following: watery or bloodshot appearance, foreign-body sensation, burning or stinging, dryness, itching, light sensitivity, blurred vision, telangiectases of the conjunctiva and eyelid margin, eyelid lid and periocular erythema, anterior blepharitis, meibomian gland dysfunction, or irregularity of eyelid margins. All patients were screened for the signs and symptoms of ocular rosacea and underwent other ophthalmologic examinations, including tear function tests. Tear functions were evaluated with Schirmer tests without anesthesia and fluorescein tear breakup time (TBUT). Tear film breakup time was assessed after instillation of $2 \%$ fluorescein staining under a cobalt blue filter. The time interval between the last complete blink and the appearance of the first dry spot was recorded. The mean of 3 consecutive measurements was obtained. The Schirmer test was performed without topical anesthesia using a standardized filter strip (Bio-Tech Vision Care). The amount of wetting was measured after 5 minutes. Meibomian gland expressibility was assessed by applying digital pressure to the eyelid margin.

Statistical Analysis-Statistical analysis of the study was performed with SPSS Statistics Version 22.0 (SPSS Inc). Continuous variables were reported as mean $(\mathrm{SD})$, and categorical variables were reported as percentages and counts. Descriptive statistics for numerical variables were created. An independent sample $t$ test was used for normally distributed continuous variables. The Kolmogorov-Smirnov test was used to determine normality. The Schirmer test without anesthesia and TBUT values among groups were compared using oneway analysis of variance. The differences were calculated using the multiple comparison Tukey test. $P<.05$ was considered statistically significant.

\section{Results}

Demographic Characteristics of Rosacea Patients-Sixty eyes of 30 newly diagnosed patients with acne rosacea with Demodex infestation and 60 eyes of 30 newly diagnosed patients with acne rosacea without Demodex infestation were enrolled in this study. The mean age (SD) of the 60 patients was 37.63 (10.01) years. The mean TBUT (SD) of the 120 eyes was 6.65 (3.44) seconds, and the mean Schirmer score (SD) was 12.59 (6.71) mm (Table 1).

Meibomian Gland Dysfunction vs Subgroup of Rosacea Patients-Thirty-four (57\%) patients had blepharitis, and 18 (30\%) patients had meibomitis. Thirty-five (58.3\%) patients had ETR, 5 (8.3\%) patients had phymatous rosacea, and $20(33.4 \%)$ patients had PPR (Table 2). Of 
the Demodex-negative patients, $73.3 \%$ (22/30) had ETR, 20\% (6/30) had PPR, and 6.7\% (2/30) had phymatous rosacea. Of the Demodex-positive patients, $43.3 \%(13 / 30)$ had ETR, 46.7\% (14/30) had PPR, and 10\% (3/30) had phymatous rosacea (Table 3 ). Papulopustular rosacea was found to be significantly associated with Demodex positivity $(P=.003)$; neither ETR nor phymatous rosacea was found to be significantly associated with Demodex infestation ( $P=.66$ and $P=.13$, respectively)(Table 3 ).

There was no statistically significant difference between the Demodex-negative and Demodex-positive groups for mean age (SD)(37.4 [11.54] years vs 37.87 [8.41] years; $P=.85)$, mean TBUT (SD)(6.73 [3.62] seconds vs 6.57 [3.33] seconds; $P=.85)$, and mean Schirmer score (SD)(13.68 [7.23] mm vs 11.5 [6.08] mm; $P=.21$ )(Table 4).

Fifteen (50\%) patients (30 eyes) in the Demodexnegative group and 19 (63.3\%) patients (38 eyes) in the Demodex-positive group had blepharitis, with no statistically significant difference between the groups $(P=.43)$. Seven $(23.3 \%$ ) patients (14 eyes) in the Demodex-negative group and $11(36.7 \%)$ patients (22 eyes) in the Demodexpositive group had meibomitis, with no statistically significant difference between the groups $(P=.39)$ (Table 3$)$.

Sixteen (53.3\%) patients (32 eyes) in the Demodexnegative group and 21 (70\%) patients (42 eyes) in the Demodex-positive group had TBUT values less than $10 \mathrm{sec}-$ onds. Eighteen (60\%) patients (36 eyes) in the Demodexnegative group and $25(83.3 \%)$ patients (50 eyes) in the Demodex-positive group had Schirmer scores less than $10 \mathrm{~mm}$ (Table 3 ). The 2 groups were not significantly different in dry eye findings $(P=.25$ and $P=.29$, respectively).

\section{Comment}

Inflammation in Rosacea-It is known that the density of nonfloral bacteria as well as $D$ folliculorum and Demodex brevis increases in skin affected by rosacea compared to normal skin. Vascular dilation associated with rosacea that results from sunlight and heat causes increased capillary permeability and creates the ideal environment for the proliferation of $D$ folliculorum. Demodex is thought to act as a vector for the activity of certain other microorganisms, particularly Bacillus oleronius,

\section{TABLE 1. Age, TBUT, and Schirmer Test Mean Values of 60 Rosacea Patients}

\begin{tabular}{lll}
$\begin{array}{l}\text { Ocular measurements } \\
\text { for rosacea }\end{array}$ & Mean no. of eyes & Mean (SD) \\
\hline Age, y & 60 & $37.63(10.01)$ \\
\hline TBUT, sec & 120 & $6.65(3.44)$ \\
\hline Schirmer score, mm & 120 & $12.59(6.71)$ \\
\hline
\end{tabular}

Abbreviation: TBUT, tear film breakup time. and thus initiates the inflammatory response associated with rosacea. ${ }^{9}$

One study reported that the inflammation associated with rosacea that was caused by Demodex and other environmental stimuli occurred through toll-like receptor 2 and various cytokines. ${ }^{10}$ It has been reported that the abnormal function of toll-like receptor 2 in the epidermis leads to the increased production of cathelicidin. Cathelicidin is an antimicrobial peptide with both vasoactive and proinflammatory activity and has been used as a basis to explain the pathogenesis of facial erythema, flushing, and telangiectasia in the context of rosacea. ${ }^{11,12}$ In addition, it has been reported that the increased secretion of proinflammatory cytokines such as IL-1 and gelatinase B in ocular rosacea leads to tearing film abnormalities that result from increased bacterial flora in the eyelids, which subsequently leads to decreased tear drainage and dry eyes. ${ }^{13}$ In addition, $B$ oleronius isolated from a $D$ folliculorum mite from patients with PPR produced proteins that induced an inflammatory immune response in $73 \%(16 / 22)$ of patients with rosacea. ${ }^{14}$

Ocular Findings in Rosacea Patients - In our study, PPR was found to be significantly associated with Demodex positivity compared to ETR and phymatous rosacea $(P=.003)$. However, ocular inflammation findings such as blepharitis and meibomitis were not significantly different between Demodex-positive and Demodex-negative patients. Although the mean Schirmer score of Demodexpositive patients was lower than Demodex-negative patients, this difference was not statistically significant. We evaluated a TBUT of less than 10 seconds and a Schirmer score less than $10 \mathrm{~mm}$ as dry eye. Accordingly, the number of patients with dry eye was higher in the Demodex-positive group, but this difference was not statistically significant.

Chronic blepharitis, conjunctival inflammation, and meibomian gland dysfunction are among the most

TABLE 2. Subgroups of Patients With Rosacea and Presence of Meibomian Gland Dysfunction $(\mathrm{N}=60)$

\begin{tabular}{ll}
\hline Rosacea subgroups/gland dysfunction & $\begin{array}{l}\text { Patients, } \\
\mathrm{n}(\%)\end{array}$ \\
\hline Blepharitis & $34(57)$ \\
Meibomitis & $18(30)$ \\
Rosacea subtype & \\
ETR & $35(58.3)$ \\
\hline Phymatous rosacea & $5(8.3)$ \\
\hline PPR & $20(33.4)$ \\
\hline
\end{tabular}

Abbreviations: ETR, erythematotelangiectatic rosacea; PPR, papulopustular rosacea. 


\section{TABLE 3. Ocular Findings for Demodex-Negative and Demodex-Positive Patients With Rosacea $(\mathrm{N}=30)$}

\begin{tabular}{llll}
$\begin{array}{l}\text { Ocular findings and subgroups } \\
\text { for Demodex }\end{array}$ & $\begin{array}{l}\text { Demodex-negative } \\
\text { patients, } \mathbf{n}(\%)\end{array}$ & $\begin{array}{l}\text { Demodex-positive } \\
\text { patients, } \mathbf{n}(\%)\end{array}$ & $\boldsymbol{P}$ value \\
\hline Blepharitis & $15(50)$ & $19(63.3)$ & .43 \\
Meibomitis & $7(23.3)$ & $11(36.7)$ & .39 \\
Rosacea subtype & $22(73.3)$ & $13(43.3)$ & .66 \\
\hline ETR & $2(6.7)$ & $3(10)$ & $.003^{\mathrm{a}}$ \\
\hline Phymatous & $6(20)$ & $14(46.7)$ & .25 \\
\hline PPR & $16(53.3)$ & $21(70)$ & .29 \\
TBUT $<10$ sec & $18(60)$ & $25(83.3)$ &
\end{tabular}

Abbreviations: ETR, erythematotelangiectatic; PPR, papulopustular rosacea; TBUT, tear film breakup time.

apapulopustular rosacea was found to be significantly associated with Demodex positivity.

TABLE 4. TBUT and Schirmer Scores for Demodex-Negative and Demodex-Positive Patients $(\mathrm{N}=30)$

\begin{tabular}{lllc}
\hline Ocular findings for Demodex & Demodex-negative patients & Demodex-positive patients & $P$ value \\
\hline Mean age (SD), y & $37.4(11.54)$ & $37.87(8.41)$ & .85 \\
\hline Mean TBUT (SD), sec & $6.73(3.62)$ & $6.57(3.33)$ & .85 \\
\hline Mean Schirmer score (SD), mm & $13.68(7.23)$ & $11.5(6.08)$ & .21 \\
\hline
\end{tabular}

Abbreviation: TBUT, tear film breakup time.

common findings of ocular rosacea. ${ }^{15,16}$ Patients with ocular rosacea commonly have dry eye and abnormal TBUT and Schirmer scores. ${ }^{17}$ In our study, we found that the fluorescein TBUT and Schirmer scores were more likely to be abnormal in the Demodex-positive group, but the difference between the 2 groups was not statistically significant.

It has been reported that proinflammatory cytokines due to a weakened immune system in rosacea patients were increased. The weakened immune system was further supported by the increased concentrations of proinflammatory cytokines such as IL-1 and matrix metalloproteinase 9 in these patients' tears and the improvement of symptoms after the inhibition of these cytokines. ${ }^{11}$ Luo et $\mathrm{al}^{18}$ reported that Demodex inflammation causes dry eye, particularly with $D$ brevis. Ayyildiz and Sezgin ${ }^{19}$ reported that Schirmer scores were significantly lower and that the Ocular Surface Disease Index had significantly increased in the Demodex-positive group compared to the Demodex-negative group ( $P=.001$ for both). A Korean study reported that Demodex density was correlated with age, sex, and TBUT results, but there was no significant relationship between Demodex density and Schirmer scores. ${ }^{16}$

Sobolewska et $\mathrm{al}^{20}$ administered ivermectin cream $1 \%$ to 10 patients with cutaneous and ocular rosacea, but only to the forehead, chin, nose, cheeks, and regions close to the eyelids, and observed a significant improvement in blepharitis $(P=.004)$. They stated that ivermectin, as applied only to the face, suppressed the proinflammatory cytokines associated with rosacea and showed antiinflammatory effects by reducing Demodex mites. ${ }^{20}$

Li et $\mathrm{al}^{21}$ demonstrated a strong correlation between ocular Demodex inflammation and serum reactivity to these bacterial proteins in patients with ocular rosacea, and they found that eyelid margin inflammation and facial rosacea correlated with reactivity to these proteins. 
These studies suggest a possible role for Demodex infestation and bacterial proteins in the etiology of rosacea.

Gonzalez-Hinojosa et $\mathrm{al}^{22}$ demonstrated that even though eyelash blepharitis was more common in PPR than ETR, there was no statistically significant association between rosacea and Demodex blepharitis. In our study, we found a significant correlation between PPR and Demodex positivity. Also, meibomian gland dysfunction was more common in the Demodex-positive group; however, this result was not statistically significant. One study compared patients with primary demodicosis and patients with rosacea with Demodex-induced blepharitis to healthy controls and found that patients with primary demodicosis and patients with rosacea did not have significantly different ocular findings. ${ }^{23}$ In contrast, Forton and De Maertelaer ${ }^{24}$ reported that patients with PPR had significantly more severe ocular manifestations compared with patients with demodicosis $(P=.004)$.

Mizuno et $\mathrm{al}^{25}$ compared the normal (nonrosacea) population with and without Demodex-infested eyelashes and found that the 2 groups were not significantly different for meibomian gland dysfunction, fluorescein TBUT, or ocular surface discomfort.

Varying results have been reported regarding the association between Demodex and blepharitis or ocular surface discomfort with or without rosacea. In our study, we found that Demodex did not affect tear function tests or meibomian gland function in patients with rosacea. We believe this study is important because it demonstrates the effects of Demodex on ocular findings in patients with cutaneous rosacea.

Limitations-Our study has some limitations. The number of patients was relatively small, resulting in few significant differences between the comparison groups. A larger prospective research study is required to assess the prevalence of Demodex mites in the ocular rosacea population along with associated symptoms and findings.

\section{Conclusion}

Rosacea is a chronic disease associated with skin and ocular manifestations that range from mild to severe, that progresses in the form of attacks, and that requires long-term follow-up and treatment. Rosacea most often presents as a disease that causes ocular surface inflammation of varying degrees. Demodex infestation may increase cutaneous or ocular inflammation in rosacea. Therefore, every patient diagnosed with rosacea should be given a dermatologic examination to determine Demodex positivity and an ophthalmologic examination to determine ocular manifestations.

\section{REFERENCES}

1. O'Reilly N, Gallagher C, Reddy Katikireddy K, et al. Demodex-associated Bacillus proteins induce an aberrant wound healing response in a corneal epithelial cell line: possible implications for corneal ulcer formation in ocular rosacea. Invest Ophthalmol Vis Sci. 2012;53:3250-3259.

2. Webster G, Schaller M. Ocular rosacea: a dermatologic perspective. J Am Acad Dermatol. 2013;69(6 suppl 1):S42-S43.
3. Crawford GH, Pelle MT, James WD. Rosacea: I. etiology, pathogenesis, and subtype classification. J Am Acad Dermatol. 2004;51:327-341.

4. Wilkin J, Dahl M, Detmar M, et al. Standard grading system for rosacea: report of the National Rosacea Society Expert Committee on the classification and staging of rosacea. J Am Acad Dermatol. 2004;50:907-912.

5. Gallo RL, Granstein RD, Kang S, et al. Standard classification and pathophysiology of rosacea: the 2017 update by the National Rosacea Society Expert Committee. J Am Acad Dermatol. 2018; 78:148-155.

6. Gao YY, Di Pascuale MA, Li W, et al. High prevalence of Demodex in eyelashes with cylindrical dandruff. Invest Ophthalmol Vis Sci. 2005;46:3089-3094.

7. Fallen RS, Gooderham M. Rosacea: update on management and emerging therapies. Skin Therapy Lett. 2012;17:1-4.

8. Erbagc1 Z, Ozgoztası O. The significance of Demodex folliculorum density in rosacea. Int J Dermatol. 1998;37:421-425.

9. Ahn CS, Huang WW. Rosacea pathogenesis. Dermatol Clin. 2018;36:81-86.

10. Forton FMN, De Maertelaer V. Two consecutive standardized skin surface biopsies: an improved sampling method to evaluate Demodex density as a diagnostic tool for rosacea and demodicosis. Acta Derm Venereol. 2017;97:242-248.

11. Yamasaki K, Kanada K, Macleod DT, et al. TLR2 expression is increased in rosacea and stimulates enhanced serine protease production by keratinocytes. J Invest Dermatol. 2011;131:688-697.

12. Gold LM, Draelos ZD. New and emerging treatments for rosacea. Am J Clin Dermatol. 2015;16:457-461.

13. Two AM, Del Rosso JQ. Kallikrein 5-mediated inflammation in rosacea: clinically relevant correlations with acute and chronic manifestations in rosacea and how individual treatments may provide therapeutic benefit. J Clin Aesthet Dermatol. 2014;7:20-25.

14. Lacey N, Delaney S, Kavanagh K, et al. Mite-related bacterial antigens stimulate inflammatory cells in rosacea. $\mathrm{Br} \mathrm{J}$ Dermatol. 2007;157:474-481.

15. Forton F, Germaux MA, Brasseur T, et al. Demodicosis and rosacea: epidemiology and significance in daily dermatologic practice. J Am Acad Dermatol. 2005;52:74-87.

16. Lee SH, Chun YS, Kim JH, et al. The relationship between Demodex and ocular discomfort. Invest Ophthalmol Vis Sci. 2010; 51:2906-2911.

17. Awais M, Anwar MI, Ilfikhar R, et al. Rosacea-the ophthalmic perspective. Cutan Ocul Toxicol. 2015;34:161-166.

18. Luo X, Li J, Chen C, et al. Ocular demodicosis as a potential cause of ocular surface inflammation. Cornea. 2017;36(suppl 1):S9-S14.

19. Ayyildiz T, Sezgin FM. The effect of ocular Demodex colonization on Schirmer test and OSDI scores in newly diagnosed dry eye patients. Eye Contact Lens. 2020;46(suppl 1):S39-S41.

20. Sobolewska B, Doycheva D, Deuter CM, et al. Efficacy of topical ivermectin for the treatment of cutaneous and ocular rosacea [published online April 7, 2020]. Ocul Immunol Inflamm. doi:10.1080/09273948.2020.1727531

21. Li J, O'Reilly N, Sheha $\mathrm{H}$, et al. Correlation between ocular Demodex infestation and serum immunoreactivity to Bacillus proteins in patients with facial rosacea. 2010;117:870-877.

22. Gonzalez-Hinojosa D, Jaime-Villalonga A, Aguilar-Montes G, et al. Demodex and rosacea: is there a relationship? Indian J Ophthalmol. 2018;66:36-38.

23. Sarac G, Cankaya C, Ozcan $\mathrm{KN}$, et al. Increased frequency of Demodex blepharitis in rosacea and facial demodicosis patients. J Cosmet Dermatol. 2020;19:1260-1265.

24. Forton FMN, De Maertelaer V. Rosacea and demodicosis: littleknown diagnostic signs and symptoms. Acta Derm Venereol. 2019; 99:47-52.

25. Mizuno M, Kawashima M, Uchino M, et al. Demodex-mite infestation in cilia and its association with ocular surface parameters in Japanese volunteers. Eye Contact Lens. 2020;46:291-296. 